\title{
Application of Deep Neural Network in Finding of Repulsive Part of Molecular Potential Based on Dispersed Emission Spectra
}

\author{
P. Gosz ${ }^{a}$, M. Krośnicki ${ }^{a}$, J. Koperski ${ }^{b}$ And T. URBAŃCZYK ${ }^{b, *}$ \\ ${ }^{a}$ Institute of Theoretical Physics and Astrophysics, University of Gdańsk, \\ Wita Stwosza 57, 80-308 Gdańsk, Poland \\ ${ }^{b}$ Smoluchowski Institute of Physics, Jagiellonian University, \\ Łojasiewicza 11, 30-348 Kraków, Poland
}

Received: 18.11.2021 \& Accepted: 17.12.2021

Doi: 10.12693/APhysPolA.141.81

*e-mail: tomek.urbanczyk@uj.edu.pl

\begin{abstract}
A new method of obtaining parameters of the repulsive part of the analytical potential energy curve of a diatomic molecule, based on the deep neural network approach, is presented. The method was successfully tested on artificially created spectra as well as an experimental bound $\rightarrow$ free spectrum of the $G 0_{u}^{+}\left(6^{1} P_{1}\right)\left(v^{\prime}=39\right) \rightarrow X 0_{g}^{+}$transition in $\mathrm{Hg}_{2}$. The method was tested for a Morse and fourparameter expanded Morse oscillator potentials but can be easily adaptable to other forms of analytical potential energy curves.
\end{abstract}

topics: deep neural network, dispersed emission spectra, potential energy curve parameters

\section{Introduction}

Nowadays, artificial neural networks and especially deep neural networks (DNN) gain increasing popularity in many applications from driving autonomous cars [1] to the synthesis of photorealistic images [2]. The neural networks are commonly used in scientific research, e.g. in astronomy for detecting gravitational lenses [3] or in particle physics for searching exotic particles [4]. This technique is also used in physical chemistry, e.g. for finding potential energy surfaces (PES) of simple molecules (e.g. $\mathrm{NH}_{2}^{+}$) based on ab-initio energy points [5] or for finding potential energy curve (PEC) of a diatomic molecule below the dissociation limit based on the so-called bound $\leftarrow$ bound experimental spectra $[6,7]$. In this work, we present an application of deep neural network (DNN) for obtaining a repulsive part of PEC (i.e., the part of PEC located above the dissociation limit) of a diatomic molecule based on bound $\rightarrow$ free experimental spectra.

Generally, there are two types of spectra that can be observed for diatomic molecules as a result of transitions between different electronic states (see Fig. 1), i.e., laser-induced fluorescence (LIF) excitation spectra and LIF dispersed emission spectra [8, 9]. In LIF excitation spectroscopy, the laser that excites molecules from a lower (usually, ground) to an upper (excited) state is gradually tuned, and the spectrum is registered as the total fluorescence of the excited molecules as a function of the laser wavelength. The total fluorescence is registered by the detector (usually a photomultiplier), which means that the spectral distribution of the fluorescence is not taken into account. This method is used to obtain so-called bound $\leftarrow$ bound spectra, which are especially useful for obtaining the PEC shape of the upper molecular state below the dissociation limit. In LIF dispersed emission spectroscopy, the wavelength of the excitation laser is fixed (it is adjusted to the energy of the chosen rotational-vibrational transition in the molecule), whereas the emitted fluorescence is registered by the spectrometer equipped with a detector. The dispersed emission spectrum shows the spectral distribution of the observed fluorescence. This experimental technique can be used for registration of the so-called bound $\rightarrow$ free spectra which are useful for obtaining characteristics of the repulsive part of PEC of the lower state involved in the investigated transition, which can not be obtained by other methods.

The bound $\rightarrow$ free LIF dispersed emission spectrum can be simulated using the BCONT program [10], which — as an input - requires parameters of the lower and upper electronic states of the studied transition. Generally, the shape of bound $\rightarrow$ free LIF dispersed emission spectrum depends on two factors: $v^{\prime}$-level wavefunction of molecule in the upper (excited) state and the shape of lower-state PEC above the dissociation limit (i.e., above the atomic asymptote with which the lower state correlates). The shape of the $v^{\prime}$-level wavefunction in the upper state is determined by the 

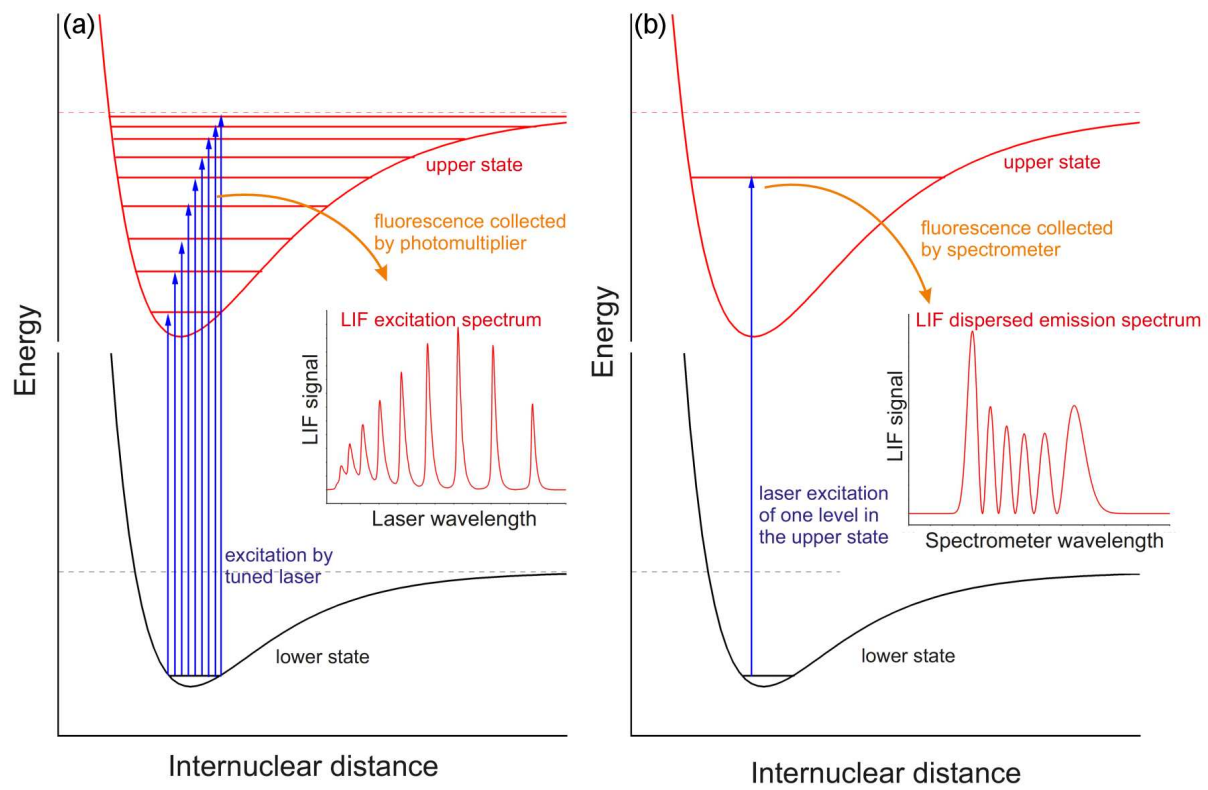

Fig. 1. The scheme of collection of (a) LIF excitation and (b) LIF dispersed emission spectra. Details in text.

upper-state PEC. The PEC of the upper state is usually known (it can be obtained, e.g. from the analysis of bound $\leftarrow$ bound LIF excitation spectra). In this case, the shape of the repulsive part of PEC in the lower state can be determined from the analysis of bound $\rightarrow$ free LIF dispersed emission spectrum.

To obtain the molecular potential below the dissociation limit from the experimental data one can use, e.g. Rydberg-Klein-Rees (RKR) method [11, 12] (which, however, is error-prone in the case of shallow potentials) or the inverted perturbation approach (IPA) methodology [13]. On the other hand, the repulsive part of the potential can be obtained from bound $\rightarrow$ free spectra, e.g. using the RKR-like inversion procedure [14]. The results of such an analysis for the ground state of $\mathrm{Hg}_{2}$ molecule are presented in $[15,16]$. The repulsive part of the potential can be also obtained using the IPA method [17]. Obtaining the whole PEC based on both bound $\rightarrow$ bound and bound $\rightarrow$ free spectra is more a challenging task. An interesting example of such an analysis for the $a^{3} \Sigma_{u}^{+}$state in $\mathrm{Na}_{2}$ molecule is presented in [18]. Moreover, having high-quality experimental data, there is also possible to determine the transition dipole moment function $M(R)$ which can be found in [18] and [19] for transitions $2^{3} \Sigma_{g}^{+}-a^{3} \Sigma_{u}^{+}$in $\mathrm{Na}_{2}$ and $4^{3} \Sigma^{+}-a^{3} \Sigma^{+}$in $\mathrm{NaK}$, respectively.

In recent years, among others our group proposed methods of obtaining the internuclear potential of dimers below the dissociation limit using artificial intelligence algorithms, i.e., neural networks $[6,7]$ and genetic algorithms [20]. Other groups reported similar approaches [21, 22]. The method of obtaining the repulsive part of the internuclear potential using DNN presented in this paper constitutes a complement of our previous study devoted to the determination of the shape of the internuclear potential well below the dissociation limit using experimental data available [6].

\section{Data preparation}

The goal of this study is to check whether the DNN can be used to find parameters of analytical PEC of the lower state which would lead to a proper simulation of experimental spectrum obtained using of the BCONT program. As an application, we examined the $G 0_{u}^{+}\left(6^{1} P_{1}\right)\left(v^{\prime}=39\right) \rightarrow X 0_{g}^{+}$transition in $\mathrm{Hg}_{2}$ molecule [16], which LIF dispersed emission spectra are presented in Fig. 2. The presented spectra contain bound $\rightarrow$ free and bound $\rightarrow$ bound transitions. In the LIF dispersed emission spectroscopy the boundary between bound $\rightarrow$ free and bound $\rightarrow$ bound part of the spectrum is determined by the difference between the energy of vibrational level in the upper state to which the molecule is excited and the energy of the asymptote of the lower state. In case of the examined transition in $\mathrm{Hg}_{2}$ this boundary is located at $48853.7 \mathrm{~cm}^{-1}$, which is marked by the blue dashed vertical line in Fig. 2 . In this work, we analyse only the bound $\rightarrow$ free part, which is located on the left-hand side of the vertical line.

Every bound $\rightarrow$ free LIF dispersed emission spectrum contains $v^{\prime}+1$ maxima, where $v^{\prime}$ is the vibrational number of the level in the upper state (in the studied example $v^{\prime}=39$ ), to which molecules are excited. However, the spectral resolution of detection of the experimental spectra sometimes is too low to resolve all maxima (especially in the highenergy part of the spectrum), which can be easily seen in Fig. 2 as trace (I). 


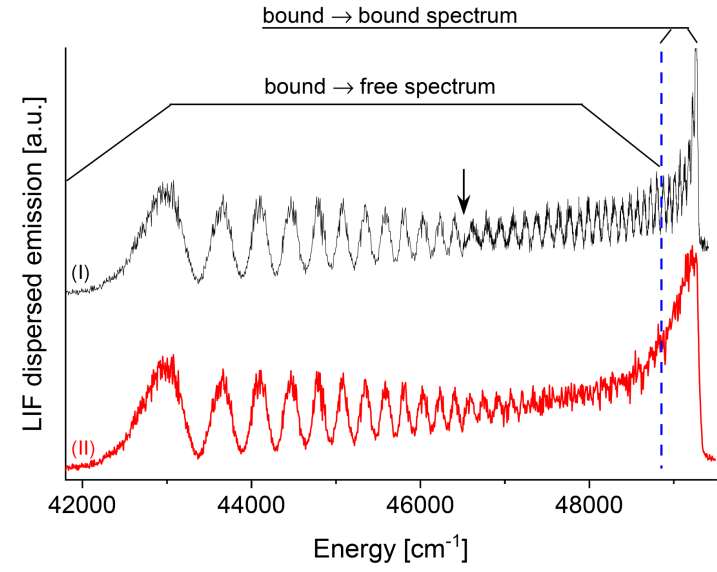

Fig. 2. The experimental LIF dispersed emission spectra of the $G 0_{u}^{+}\left(v^{\prime}=39\right) \rightarrow X 0_{g}^{+}$transition in $\mathrm{Hg}_{2}$ [16] analysed in this work. (I) Low resolution spectrum recorded with $100 \mathrm{~cm}^{-1}$ monochromator slit-width. (II) Higher-resolution spectrum combined with: low resolution spectrum from (I) and higher resolution spectrum recorded with monochromator slit-width $10 \mathrm{~cm}^{-1}$. The vertical arrow denotes boundary between low- and higherresolution parts. The dashed blue vertical line shows boundary between bound $\rightarrow$ free and bound $\rightarrow$ bound parts of the spectrum. Details in text.

In the studied example, assuming that the $G 0_{u}^{+}$ state potential is known, the positions of the observed maxima depend on parameters of the $X 0_{g}^{+}$ state potential. For a given set of potential parameters of the $X 0_{g}^{+}$state, the simulated positions of maxima in the emission spectrum can be easily found using BCONT program. In this work, we assumed that the upper-state PEC is described by the pointwise ab-initio potential of [23]. We also assumed, that the repulsive part of the $X 0_{g}^{+}$state potential is described by the expanded Morse oscillator (EMO) function [24]

$$
V_{\mathrm{EMO}}(R)=D_{e}\left[\left(\mathrm{e}^{-\beta(R)\left(R-R_{e}\right)}-1\right)^{2}-1\right] .
$$

This EMO function has three parameters: potential well depth $D_{e}$, internuclear equilibrium distance $R_{e}$ and $\beta(R)$ which determines the "width" of the potential well. It is defined as power series

$\beta(R)=\beta_{0}+\beta_{1} \frac{R-R_{\mathrm{ref}}}{R+R_{\mathrm{ref}}}+\beta_{2}\left(\frac{R-R_{\mathrm{ref}}}{R+R_{\mathrm{ref}}}\right)^{2}+\ldots$,

where $R_{\text {ref }}$ is the expansion centre usually (also in this work) set to $R_{e}$. If the series is cut after first term $\left(\beta(R)=\beta_{0}\right)$, the EMO potential is reduced to a Morse potential

$$
V_{\text {Morse }}(R)=D_{e}\left[\left(\mathrm{e}^{-\beta\left(R-R_{e}\right)}-1\right)^{2}-1\right] .
$$

Here we represent the $X 0_{g}^{+}$state potential by the Morse potential (which should be sufficient to simulate a low-resolution experimental spectrum with fewer resolved maxima presented in Fig. 2 by (I)) or
TABLE I

Parameters of Morse and four-parameter EMO representations of the $\mathrm{X}_{g}^{+}$state in $\mathrm{Hg}_{2}$ obtained by the DNN. Details in text.

\begin{tabular}{c|c|c|c}
\hline \hline Parameter & Range* $^{*}$ & $\begin{array}{c}\text { Morse } \\
\text { potential }\end{array}$ & $\begin{array}{c}\text { Four-parameter } \\
\text { EMO potential }\end{array}$ \\
\hline$R_{e}[\AA]$ & $3.5-3.75$ & 3.498 & 3.449 \\
$D_{e}\left[\mathrm{~cm}^{-1}\right]$ & $365-385$ & 376.62 & 374.25 \\
$\beta_{0}\left[\AA^{-1}\right]$ & $1.2-1.45$ & 1.4833 & 1.5535 \\
$\beta_{1}\left[\AA^{-1}\right]$ & $0-0.03$ & - & $6.44 \times 10^{-4}$ \\
\hline
\end{tabular}

${ }^{*}$ The range from which parameters are randomly

chosen during creation of training data set.

the EMO potential with only two non-zero $\beta$ terms $\left(\beta_{i>2}=0\right.$ in (1)), which was used to simulate the spectrum collected with higher resolution (trace (II) in Fig. 2). As the version of EMO potential used in this work has four parameters $\left(R_{e}, D_{e}, \beta_{0}\right.$ and $\left.\beta_{1}\right)$, in the further part of the article it will be compendiously named as four-parameter EMO potential.

Generally, based on the positions of experimental maxima, the trained DNN should return parameters of the $X 0_{g}^{+}$state potential which lead to the BCONT simulation being in agreement with the experimental spectrum. Based on the previous experimental results as well as of ab-initio calculations, for each potential parameter of the $X 0_{g}^{+}$state, we defined a range in which the parameter value is probably located. The chosen ranges are presented in Table I.

To train the DNN for both network architectures (first, designed for obtaining parameters of a Morse potential and second, for obtaining parameters of a four-parameter EMO potential, more details in Sect. 3), we generated the data sets containing over 10000 data samples which served as training and validation sets. In the data sets, each sample contained two vectors. Namely,

1. The output vector $\boldsymbol{y}_{i}=\left(R_{e}^{i}, D_{e}^{i}, \beta_{0}^{i}\right)$ for DNN predicting parameters of a Morse potential or $\boldsymbol{y}_{i}=\left(R_{e}^{i}, D_{e}^{i}, \beta_{0}^{i}, \beta_{1}^{i}\right)$ for the DNN predicting parameters of a four-parameter EMO potential.

2. The input vector $\boldsymbol{x}_{i}$ which contained the differences between energies of the first $N$ maxima observed in the BCONT simulation of the $G 0_{u}^{+}\left(6^{1} P_{1}\right)\left(v^{\prime}=39\right) \rightarrow X 0_{g}^{+}$transition based on the $X 0_{g}^{+}$state potential with parameters from $\boldsymbol{y}_{i}$ and the energy of the first maximum observed in the simulation. It means that $j$-th component of the $\boldsymbol{x}_{i}$ vector $x_{i}^{j}=$ $E_{i}^{s i m_{j}}-E_{i}^{s i m_{0}}$, where $E_{i}^{s i m_{j}}$ and $E_{i}^{s i m_{0}}$, denotes energies of $j$-th and the first maximum observed in the BCONT simulation based on the $X 0_{g}^{+}$state potential with the parameters collected in $\boldsymbol{y}_{i}$ vector. 
Using differences between the energies of subsequent maxima and the energy of the first maximum (instead of the energies of subsequent maxima directly) is due to the fact that the experimental spectrum can be shifted along the $x$ horizontal axis due to limitation in measurement of absolute energies in the spectrum. To compare the BCONT simulation and experimental spectrum, the simulation is shifted to obtain agreement between the energy of the first experimental maximum and the first maximum in the simulation.

Vectors $\left(\boldsymbol{x}_{i}, \boldsymbol{y}_{i}\right)$ consist of single vector pair used in the process of training DNN. For each sample in the data set, the values of potential parameters $\left(\boldsymbol{y}_{i}\right)$ were randomly chosen with uniform distribution from defined ranges (compare with the second column of Table I). The value of $N$ was chosen based on the number of maxima observed in the experimental spectrum. In the case of the DNN which predicts parameters of a Morse potential, we considered energies of the first 15 maxima $(N=15)$, whereas in the case of DNN predicting parameters of a four-parameter EMO potential, the energies of the first 25 maxima were considered $(N=25)$.

\section{Neural network architecture}

The DNN was created in Python programming language using TensorFlow library of [25] with Keras API of [26]. The network was trained using cloud environment Google Colaboratory [27], however it can also be trained or used on the local computer. We prepared two architectures of DNN with regard to potential type of the $X 0_{g}^{+}$state. To obtain parameters of a Morse potential, we used fully

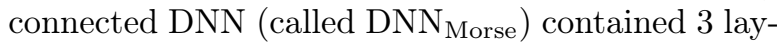
ers with 25, 10 and 3 neurons in subsequent layers. To obtain parameters of four-parameter EMO potential, the fully connected DNN (called DNN $\mathrm{EMO}$ ) with 6 layers was used (with 60, 60, 100, 100, 60 and 4 neurons in subsequent layers). The training sets contained 12000 and 15000 samples for DNN $\mathrm{DNorse}_{\text {}}$ and $\mathrm{DNN}_{\mathrm{EMO}}$, respectively.

In both architectures, all layers had LeakyReLU activation function with default value of negative slope coefficient $\alpha=0.3$. The DNNs used ADAM optimizer [28] with learning rate set to 0.008 , the weights were initialized using variance scaling initializer. The maximum number of epochs was set to 2500 , however further described early stopping mechanism was also used. During learning process the cost functions were calculated as the mean absolute percentage error. The training data were normalized using normalization module of the Keras library. For both networks, $10 \%$ of datasamples from the training data sets were used as the validation data set. Thanks to this approach we implemented two improvements:

1. If the cost function for validation data set did not decrease during 25 epochs, the learning rate was reduced by multiplying it by 0.8 .
2 . If the cost function for validation data set decreased less then 0.0005 during 180 epochs, the early stopping condition occurred, which meant that the training process was ceased and the weights associated with smallest value of cost function (for validation data set) were restored.

In Google Colaboratory environment the training of DNN took usually 1-2 min in case of $\mathrm{DNN}_{\text {Morse }}$ and 5-7 min in case of $\mathrm{DNN}_{\mathrm{EMO}}$. Using a local computer (Intel(R) Core(TM) i5-1135G7 processor, 16GB of RAM, Nvidia GeForce MX 450) the same processes took about 3-4 min for $\mathrm{DNN}_{\text {Morse }}$ and 6-10 min for $\mathrm{DNN}_{\mathrm{EMO}}$.

\section{Results}

\subsection{Tests on artificially generated spectra}

The main goal of training the DNN was to find the repulsive part of PEC of the $\mathrm{X}_{\mathrm{g}}^{+}$state in $\mathrm{Hg}_{2}$ which lead to the proper simulation of the experimental spectrum of the $G 0_{u}^{+} \rightarrow X 0_{g}^{+}$transition. However, to verify the correctness and versatility of the proposed method, we also conducted tests on artificially generated spectra (reference spectra). This idea is based on the fact that knowing PEC of the repulsive part of the $X 0_{g}^{+}$state (i.e., potential parameters), it is very easy to obtain the simulated spectrum of the $G 0_{u}^{+} \rightarrow X 0_{g}^{+}$transition using the BCONT program. To test the DNN one can randomly choose parameters of the $\mathrm{X}_{g}^{+}$state PEC, next, perform BCONT simulation based on these parameters, and use the simulated spectrum (precisely, the energies of maxima obtained from the spectrum) to test the DNN.

Let us assume that $E_{i}^{\mathrm{DNN}}$ and $E_{i}^{\mathrm{ref}}$ are energies of the position of the $i$-th maximum in the spectrum simulated based on the DNN result and in the reference spectrum, respectively. Therefore, we can introduce metric $C$ which evaluates the agreement between simulation based on the DNN result and the reference spectrum. It is defined as

$$
C=\frac{1}{N} \sum_{i=1}^{N}\left|\left(E_{i}^{\mathrm{ref}}-E_{0}^{\mathrm{ref}}\right)-\left(E_{i}^{\mathrm{DNN}}-E_{0}^{\mathrm{DNN}}\right)\right|
$$

where $N$ is the number of considered maxima in the spectrum. It is obvious that in case of "a perfect simulation" ( $E_{i}^{\mathrm{ref}}=E_{i}^{\mathrm{DNN}}$ for each maximum) $C$ is equal zero.

For both DNN architectures we created new data sets containing 1000 samples with PEC parameters chosen randomly from the same ranges as in the case of training data sets. Table II collects the results of performed tests, whereas Fig. 3 shows comparison between simulations based on DNN results and reference spectra for samples for which $C$ parameter is similar to average $C$ in the whole data set. Figure 4 presents the distribution of $C$ for the test data sets for both DNN architectures. As one can see, the 


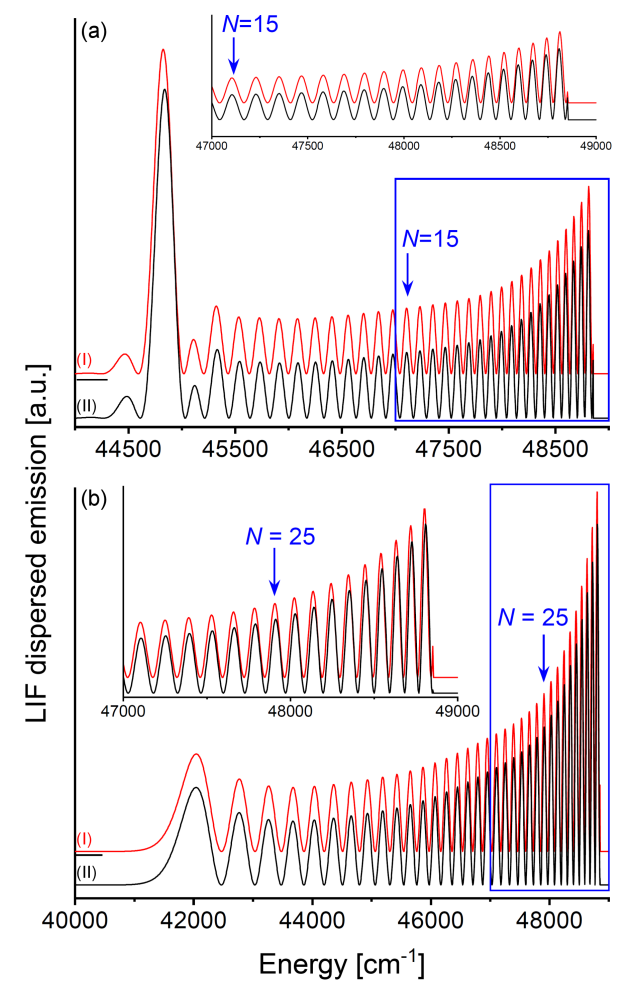

Fig. 3. Comparison between (I) artificially created reference spectra from test data sets and (II) simulation based on DNN result. Panels (a) and (b) present results for DNN predicting parameters of a Morse potential and four-parameter EMO potential, respectively. Presented examples have agreement metrics $C$ similar to an average $C$ in test data set for DNN predicting particular type of potential ((a) $12.8 \mathrm{~cm}^{-1}$ and (b) $46.9 \mathrm{~cm}^{-1}$ ). Insets show fragments depicted with blue rectangles and arrows indicate the last maxima, which were considered by the DNNs ( $N$ values correspond to the length of input vectors of DNNs). Details in text.

averaged $C$ parameter for $\mathrm{DNN}_{\text {Morse }}$ is significantly lower than that for $\mathrm{DNN}_{\mathrm{EMO}}$. This is due to the fact that predicting parameters of four-parameter EMO potential is more challenging than for a Morse potential, which has only three parameters. However, for both analysed forms of potential the parameters predicted by DNNs lead to BCONT simulation with satisfactory agreement with the relevant reference spectrum, which is depicted in Fig. 3.

\subsection{Simulation of experimental spectra}

LIF dispersed emission spectrum of the $\mathrm{GO}_{u}^{+} \rightarrow$ $X 0_{g}^{+}$transition in $\mathrm{Hg}_{2}$ [16] encompasses $8000 \mathrm{~cm}^{-1}$ (i.e., $40 \mathrm{~nm}$ ). Due to the vast range of the spectrum, its low-resolution version was recorded with a larger monochromator step (distance between neighbouring points along the horizontal axis) $\delta=7.2 \mathrm{~cm}^{-1}$ and the monochromator slit-width was set to $100 \mathrm{~cm}^{-1}$. The spectrum is shown in Fig. 5a (trace $(\mathrm{I}))$. To resolve maxima in the

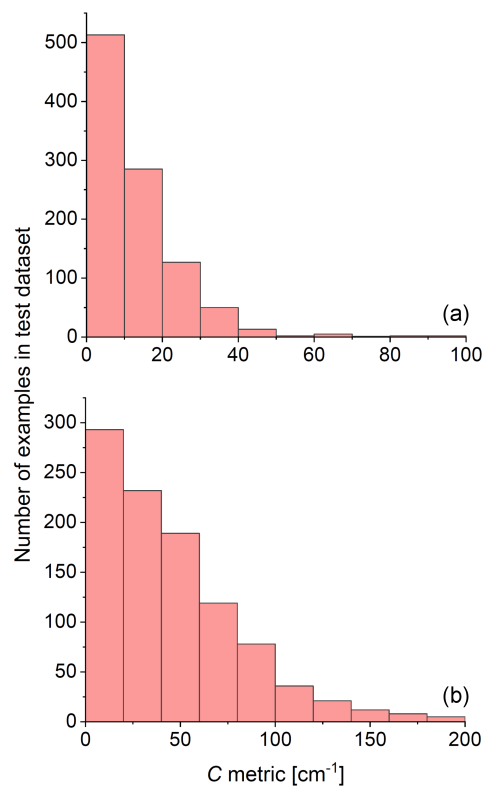

Fig. 4. Distribution of $C$ metric parameter which measures agreement between simulation based on DNN result and reference spectrum for the test data sets. Panel (a) and (b) show results for $\mathrm{DNN}_{\text {Morse }}$ and $\mathrm{DNN}_{\mathrm{EMO}}$, respectively. Details in text.

TABLE II

Analysis of agreement between reference spectra and their simulations based on DNN results measured by $C$ metric parameter (compare with (4)). Statistics for the test data set (not used to train the DNN) containing 1000 samples. Details in text.

\begin{tabular}{l|c|c|c}
\hline \hline & $\begin{array}{c}\text { Average } \\
{\left[\mathrm{cm}^{-1}\right]}\end{array}$ & $\begin{array}{c}\text { Median } \\
{\left[\mathrm{cm}^{-1}\right]}\end{array}$ & $\begin{array}{c}\text { Std. dev. } \\
{\left[\mathrm{cm}^{-1}\right]}\end{array}$ \\
\hline $\mathrm{DNN}_{\text {Morse }}(\mathrm{N}=15)$ & 12.8 & 9.7 & 11.7 \\
$\mathrm{DNN}_{\text {EMO }}(\mathrm{N}=25)$ & 46.9 & 37.2 & 39.7
\end{tabular}

high-energy part of the spectrum (which are not resolved in the low-resolution spectrum), the part of the spectrum for energies above $46500 \mathrm{~cm}^{-1}$ was recorded with higher resolution $\left(\delta=2.4 \mathrm{~cm}^{-1}\right.$, $20 \mathrm{~cm}^{-1}$ slit-width).

It is worth mentioning that only the high-energy part of the spectrum was recorded with higher resolution due to the fact that high-resolution measurements are significantly more time consuming than low-resolution ones. Traces marked by (I) in Fig. $5 \mathrm{~b}$ and c present the experimental spectrum combined with the low- and higher-resolution parts for energies smaller and larger than $46500 \mathrm{~cm}^{-1}$, respectively.

Simulation based on a Morse potential with parameters obtained by $\mathrm{DNN}_{\text {Morse }}$ is shown as trace (II) in Fig. 5a and b. To simulate the influence of different monochromator slit-widths, the simulated spectrum was convolved with a Gaussian 


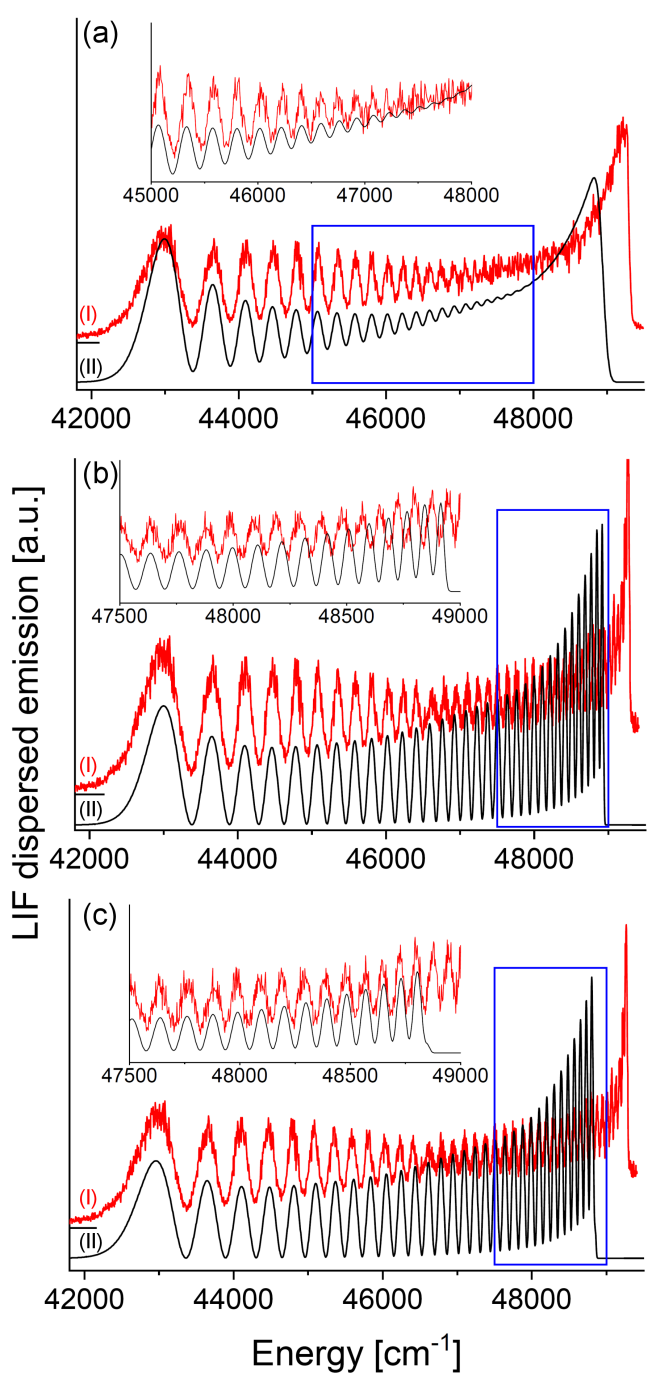

Fig. 5. (I) Experimental spectra and (II) their simulations based on the DNN results of the $G 0_{u}^{+}\left(6^{1} P_{1}\right)\left(v^{\prime}=39\right) \rightarrow X 0_{g}^{+}$transition in $\mathrm{Hg}_{2}$. Trace (I) in panel (a) shows low-resolution experimental spectrum recorded with $100 \mathrm{~cm}^{-1}$ monochromator slit-width whereas traces (I) in panel (b) and (c) present combination of low- and higher-resolution spectra recorded with $100 \mathrm{~cm}^{-1}$ and $20 \mathrm{~cm}^{-1}$ monochromator slit-widths, respectively. The simulation (traces (II) presented in panel (b) and (c)) is based on a Morse potential with parameters predicted by $\mathrm{DNN}_{\text {Morse}}$, whereas simulation in panel (c) is based on four-parameter EMO potential with parameters predicting by DNNEMO. Boundary of insets are depicted with blue rectangles. Values of parameters of both potentials predicted by DNNs are collected in Table I. Details in text.

function with different $\sigma$ parameters, i.e., $\sigma=60 \mathrm{~cm}^{-1}$ and $\sigma=10 \mathrm{~cm}^{-1}$ in Fig. 5a and $b$, respectively. One can see that the simulation is in agreement with the low-resolution spectrum (Fig. 5a), whereas in the case of the higher-resolution spectrum (inset in Fig. 5b) there are significant disagreements between simulation and experimental spectrum. The result of simulation based on four-parameter EMO potential with parameters predicted by $\mathrm{DNN}_{\mathrm{EMO}}$ is presented as (II) in Fig. 5c. In this case, the simulation is in agreement with the experimental data also in the higher-resolution part of the spectrum. It is also important to emphasize that the simulation presented in this work should be compared with the experimental spectrum only with respect to position of minima and maxima, and not comparing intensities of maxima. The experimental spectrum consists of two parts, which were recorded using different spectrometer settings (especially, the entrance slit) and, afterwards, were manually combined into one spectrum. Due to the procedure, the simulated intensities of maxima may differ from the observed ones.

It should be also stressed that the simulation presented in Fig. 5 refers only to the bound $\rightarrow$ free part of the spectrum. The experimental spectrum contains also the bound $\rightarrow$ bound component in its highfrequency part, which was not considered in the simulation. The simulation of bound $\rightarrow$ bound part of the spectrum, which can be done using e.g. LEVEL program [29], depends on the part of PEC below the dissociation limit. We envision that there is a possibility of designing DNN method, which can determine the whole PEC based on both bound $\rightarrow$ bound and bound $\rightarrow$ free spectra. This problem is complicated, however, we are going to explore it in the future.

\section{Conclusions}

The test results conducted for artificially created test data sets show that the potential parameters predicted by both $\mathrm{DNN}_{\text {Morse }}$ and $\mathrm{DNN}_{\mathrm{EMO}}$ can lead to $\mathrm{CO}$ simulation the consistent with corresponding reference spectra (compare with Fig. 3). Moreover, simulations of the $G 0_{u}^{+}\left(v^{\prime}=39\right) \rightarrow X 0_{g}^{+}$transition in $\mathrm{Hg}_{2}$ based on potential parameters predicted by both DNNs also show a good agreement with the experimental spectra (compare with Fig. 5). This indicates that the presented method can be used for obtaining the repulsive part of PEC above its dissociation-energy limit based on LIF dispersed emission spectra. The presented method can be easily adapted to other forms of analytical PEC, e.g. Lennard--Jones or van der Waals potentials.

The dispersed emission spectrum depends on both lower and upper electronic state potentials involved in the observed transition. In this analysis, we used a newer, more precise representation of the potential of the $G 0_{u}^{+}$state obtained using IPA method [23], whereas the analysis presented in [16] based on the previous $G 0_{u}^{+}$state representation obtained using RKR-method. Due to this fact, a direct comparison of the potential energy curve of the $X 0_{g}^{+}$ state obtained in this work with the curve of [16] is difficult. Consequently, the most reliable method of 
assessing the quality of the determination of the repulsive part of the $X 0_{g}^{+}$state potential in $\mathrm{Hg}_{2}$ using DNN is the comparison of the simulation based on the obtained $X 0_{g}^{+}$state potential with the experimental spectrum (see Fig. 5).

It is also important to emphasize that the method presented in this work is an alternative approach to the problem of determining the repulsive part of diatomic molecule potential. We do not claim the presented method is better than the respected RKR-like inversion procedure [14], but we show the possibility of using neural networks to find the repulsive parts of potential energy curves using dispersed emission spectra. In the future, we are going to develop a method that can by employed to obtain the whole molecular potential based on both bound $\leftarrow$ free and bound $\leftarrow$ bound spectra.

\section{References}

[1] J. Kocic, N. Jovicic, V. Drndarevic, Sensors 19, 2064 (2019).

[2] T. Karras, S. Laine, M. Aittala, J. Hellsten, J. Lehtinen, T. Aila, Analyzing and improving the image quality of stylegan, in: 2020 IEEE/CVF Conf. on Computer Vision and Pattern Recognition (CVPR), Seattle (WA), IEEE, 2020, p. 8107.

[3] C. Schaefer, M. Geiger, T. Kuntzer, J.-P. Kneib, Astron. Astrophys. 611, A2 (2018).

[4] P. Baldi, P. Sadowski, D. Whiteson, Nat. Commun. 5, 4308 (2014).

[5] Z. Yang, S. Wang, J. Yuan, M. Chen, Phys. Chem. Chem. Phys. 21, 22203 (2019).

[6] D. Horwat, M. Krosnicki, T. Urbanczyk, J. Koperski, Mol. Simul. 47, 650 (2021).

[7] T. Urbanczyk, J. Koperski, Spectrochim. Acta A 189, 502 (2018).

[8] W. Demtröder, Laser Spectroscopy, Vol. 2, Experimental Techniques, Springer-Verlag, Berlin 2008.

[9] P. Andresen, Optical Measurements, Ch. Laser Induced Fluorescence, SpringerVerlag, Berlin 2001, p. 199.

[10] R. LeRoy, G. Kraemer, BCONT 2.2, University of Waterloo Chemical Physics Research Report CP-650R2, 2004.

[11] S.M. Kirschner, J.K. Watson, J. Mol. Spectrosc. 47, 234 (1973).

[12] R.J. Le Roy, J. Quant. Spectrosc. Radiat. Transf. 186, 158 (2017).
[13] A. Pashov, W. Jastrzebski, P. Kowalczyk, Comput. Phys. Commun. 128, 622 (2000).

[14] M. S. Child, H. Essén, R.J. Le Roy, J. Chem. Phys. 78, 6732 (1983).

[15] J. Koperski, Phys. Rep. 369, 177 (2002).

[16] J. Koperski, J. Atkinson, L. Krause, J. Mol. Spectrosc. 184, 300 (1997).

[17] V.S. Ivanov, V.B. Sovkov, L. Li, A.M. Lyyra, T.J. Whang, S. Magnier, J. Chem. Phys. 114, 6077 (2001).

[18] C. Faust, J. Jones, J. Huennekens, R.W. Field, J. Chem. Phys. 146, 104302 (2017).

[19] B. McGeehan, S. Ashman, C. Wolfe, R. Steinhardt, M. Monaco, J. Huennekens, A. Hickman, J. Mol. Spectrosc. 265, 74 (2011).

[20] T. Urbanczyk, J. Koperski, Mol. Simul. 46, 1073 (2020).

[21] M.M. Almeida, F.V. Prudente, C.E. Fellows, J.M.C. Marques, F.B. Pereira, J. Phys. B 44, 225102 (2011).

[22] A.C.P. Bittencourt, F.V. Prudente, J.D.M. Vianna, Chem. Phys. 297, 153 (2004).

[23] M. Krosnicki, M. Strojecki, T. Urbanczyk, A. Pashov, J. Koperski, Phys. Rep. 591, 1 (2015).

[24] J.Y. Seto, Z. Morbi, F. Charron, S.K. Lee, P.F. Bernath, R.J. Le Roy, J. Chem. Phys. 110, 11756 (1999).

[25] M. Abadi, A. Agarwal, P. Barham et al., TensorFlow: Large-scale Machine Learning on Heterogeneous Systems, 2015.

[26] F. Chollet, Qianli Scott Zhu, F. Rahman et al., Keras, 2015.

[27] E. Bisong, Google Colaboratory, in: Building Machine Learning and Deep Learning Models on Google Cloud Platform: A Comprehensive Guide for Beginners, Apress, Berkeley (CA) 2019, p. 59.

[28] D.P. Kingma, J. Ba, in: 3rd Int. Conf. on Learning Representations (ICLR 2015), Eds. Y. Bengio, Y. LeCun, arXiv:1412.6980, San Diego (CA) 2015.

[29] R.J. Le Roy, J. Quant. Spectrosc. Radiat. Transf. 186, 167 (2017). 